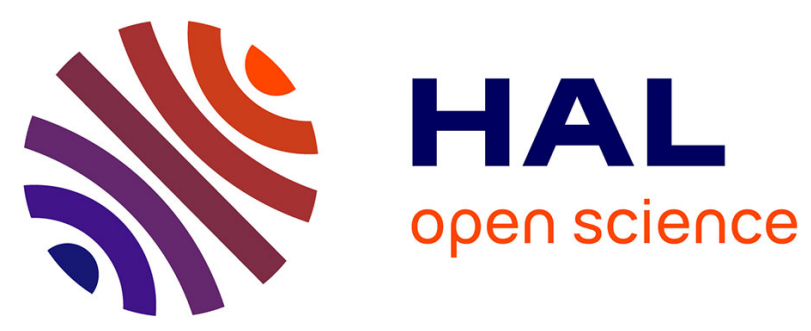

\title{
Urban Disaster Simulation Incorporating Human Psychological Models in Evacuation Behaviors
}

Tatsuya Yamazaki, Hiroyuki Tamai, Yasunori Owada, Kiyohiko Hattori, Shin'ichi Taira, Kiyoshi Hamaguchi

\section{- To cite this version:}

Tatsuya Yamazaki, Hiroyuki Tamai, Yasunori Owada, Kiyohiko Hattori, Shin'ichi Taira, et al.. Urban Disaster Simulation Incorporating Human Psychological Models in Evacuation Behaviors. 1st International Conference on Information Technology in Disaster Risk Reduction (ITDRR), Nov 2016, Sofia, Bulgaria. pp.20-30, 10.1007/978-3-319-68486-4_3 . hal-03213115

\section{HAL Id: hal-03213115 \\ https://hal.inria.fr/hal-03213115}

Submitted on 30 Apr 2021

HAL is a multi-disciplinary open access archive for the deposit and dissemination of scientific research documents, whether they are published or not. The documents may come from teaching and research institutions in France or abroad, or from public or private research centers.
L'archive ouverte pluridisciplinaire HAL, est destinée au dépôt et à la diffusion de documents scientifiques de niveau recherche, publiés ou non, émanant des établissements d'enseignement et de recherche français ou étrangers, des laboratoires publics ou privés.

\section{(c)(1)}

Distributed under a Creative Commons Attribution| 4.0 International License 


\title{
Urban Disaster Simulation Incorporating Human Psychological Models in Evacuation Behaviors
}

\author{
Tatsuya Yamazaki ${ }^{1}$, Hiroyuki Tamai ${ }^{1}$, Yasunori Owada ${ }^{2}$, Kiyohiko Hattori $^{2}$, \\ Shin'ichi Taira ${ }^{2}$, and Kiyoshi Hamaguchi ${ }^{3}$ \\ ${ }^{1}$ Princeton University, Princeton NJ 08544, USA \\ ${ }^{1}$ Graduate School of Science and Technology, Niigata University, Niigata, Japan \\ ${ }^{2}$ Resilient ICT Research Center, National Institute of Information and Communications \\ Technology, Sendai, Japan \\ ${ }^{3}$ Wireless Networks Research Center, National Institute of Information and Communications \\ Technology, Yokosuka, Japan \\ yamazakilie.niigata-u.ac. jp
}

\begin{abstract}
Building evacuation simulation provides us with various knowledge and suggestion before a real disaster happens. To date, however, evacuees were often modeled as homogeneous without individual motivation in a large-scale urban simulation model, which is rather different from real human behavior. In this paper, an evacuation simulation model with human psychological models is developed for urban disaster situation. Three psychological models are actually incorporated: normalcy bias, emotional contagion bias, and sympathy behavior bias. Normalcy bias is the initial evacuation delay caused by a belief that abnormal events rarely happen. Emotional contagion is the effect of one person's emotional state on the emotional state of people around him/her both explicitly and implicitly. Simulated experimental results show that the proposed model provides accurate evacuation behaviors than the normal behavior model without psychological consideration.
\end{abstract}

Keywords: Disaster, Evacuation, Multi-Agent System, Psychological Model.

\section{Introduction}

As one of the largest disasters, the Great East Japan Earthquake and its following tsunami are memorable, which resulted in losing about 15,900 lives and missing about 2,900 people [1]. When such disasters including not only earthquakes but also hurricanes, floods, and so on occur at a populated area, damage will become huge and tremendous. Recently, since computing power is available at a low cost and GIS (Geographic Information System) is developed, computer simulations have become a powerful tool to prepare disasters. Namely, computer simulations may enable us to find out a bottleneck point when people are evacuating or to educate people which is a better evacuation way.

MAS (Multi-Agent System) is a popular scheme to simulate a disaster situation on a computer platform. MAS contains an environment, objects, and agents. In MAS, an 
agent can be a physical or virtual entity that can act, perceive its environment (in a partial way) and communicate with others [2]. The agents are autonomous and relations between the agents as well as a set of operations that can be performed by the agents can be defined.

Agent behaviors can be physically reproduced through computer simulations using MAS. Though evacuation models built on GIS obey physical rules defined on buildings, roads, railways, etc., important psychological parameters are not frequently used [3]. In addition, evacuating agents are often modeled as homogeneous without individual motivation in a large-scale urban simulation model, which is rather different from real human behavior. Modeling psychological variables will enhance prediction of human behavior during evacuations. Apart from MAS, there is a previous research that designs and analyzed evacuation routes in transportation networks in cases of natural disasters [4]. Therein, a method for defining two independent paths from the disaster area to each destined shelters for vehicle flow allocation in evacuation planning, considering both travel time and capacity of the transportation network.

In this paper, an evacuation simulation model with human psychological models is developed for urban disaster situation. We introduce three typical psychological biases: normalcy bias, emotional contagion bias, and sympathy behavior bias.

Firstly, normalcy bias, or the normality bias, is the initial evacuation delay caused by a belief that abnormal events rarely happen. It is one of the most usual psychological biases and can be seen in case of hurricanes, typhoons, Tsunami or floods [5]. Seconary, the emotional contagion bias is the effect of one person's emotional state on the emotional state of people around him/her both explicitly and implicitly. Simply speaking, this bias is to follow to the others and following a person with leadership belongs to this category. Finally, the sympathy behavior bias means that people instinctively take care of others, especially the weak such as elderly persons, injured persons, and so on. Without considering the kindness to others that people inherently have, accurate simulation results cannot be obtained.

Vorst [3] stated as follows: "Most people like to postpone evacuation till more convincing observations are available. Leaving home is very uneasy and risky." His statement exactly tallies with what we call the normalcy bias. Also, Vorst pointed out such evacuation models that reflect the normalcy bias was not represented in simulations of evacuation procedures [3].

We propose human psychological models to realize the above biases. The proposed models have two points mainly. The first point is that a parameter is incorporated in each agent to implement the normalcy bias. The second point is that a function of recognizing the other agents is realized for each agent. Combination of the introduced parameter and the recognition function enables the agent to equip emotional $\mathrm{c}$ ontagion bias. Moreover, we introduce the role of agent such as elderly persons. The agent role in combination of the recognition function is used to realize the sympathy behavior bias.

The proposed models are evaluated by simulation experiments for urban dis-aster cases. For the experiments, agents with the proposed psychological models are implemented on a simulation system with GIS data. A virtual city area is pre-pared for the 
area where the agents walk to find out their shelters. The experimental results show that more accurate human behaviors are realized by the pro-posed model.

The rest of this paper is organized as follows. Section 2 introduces the related work and the detailed explain of the proposed models is provided in Section 3. After the simulation experiment setting is described in Section 4, the experimental results are presented in Section 5. Finally, Section 6 concludes this paper.

\section{Related Work}

Agents and MAS have been studied in the area of AI (Artificial Intelligence). In the AI area, agents are autonomous entities which observe environments through their sensors and act on the environments using their actuators. Agents are programmed to achieve their goals autonomously by interacting with the environments. When an agent has ability to change its behavior according to the results of action, it is said that the agent can learn. Reinforcement learning is a scheme of learning where agents change their behaviors according to the rewards from the environments. Reinforcement learning was often applied to the case of learning game strategies [6].

When the agent technology was applied to disaster simulations, it is simply called an agent-based simulation or system (ABS) initially. Wu et al. [7] have developed the Dynamic Discrete Disaster Simulation System (D4S2), that is a comprehensive decision support system to simulate the large-scale disaster responses. D4S2 has an interface with a GIS system and a result was visualized on a GIS map. However, the responder agents' operations were regulated by a set of rules.

Chen et al. [8] analyzed real-time cell phone data and developed Dynamic Adaptive Disaster Simulation (DADS), which is a system capable of predicting population movements in large-scale disasters. It unifies diverse modeling concepts and techniques as well as considering cell phone and GIS data. In their simulations, they could predict population movements during a disaster like Hurricane Katrina accurately. The DADS system includes two types of agents: synthetic and predictive agents. In the simulation, the synthetic agents generate a set of real-time data using the real-world cell phone data. The predictive agent is associated with a cell phone user one by one and moves to represent predictions of the cell phone user's future movements.

Yun et al. [9] proposed a swarm-based dynamic disaster evacuation simulation model. Their simulation model was run on a simulated platform for complicated modeling system called Swarm invented by the Santa Fe Institute. In their simulation, every agent had the same property but they could get better performance of evacuation by changing the evacuation command dynamically than the static evacuation plan. Samira and Fatima [10] proposed a multi-agent simulation. Their purpose was to save a heart attack victim, which is different from the disaster evacuation. Therefore, they included three different types of agents in the simulation: victim, environment, and succourer agents. Among them, the succourer agent belongs to BDI (Belief, Desire and intention) type agents and it has beliefs about the world in which it operates, and it must meet the desires by making intentions the behavior of the system, until the emergence of approaches oriented agents. But concrete implementation methods were unclear, so that 
it is difficult to apply this BDI type to the disaster evacuation cases.

More recently, Mustaphaa et al. [11] proposed an agent-based methodological framework for complex system (Supply Chain, Natural Disaster) including GIS database. The framework itself seems to be general and it reflects the organizational structure and policies within the simulation. They also proposed various models in the framework as well as a specification of the translation process between the models. No implementation of simulation models nor simulation results, however, appeared in this paper. Nevertheless, it is true that interest in human factors and psychology is growing in the area of evacuation research [12], where human factors include physical, cognitive, motivational and social variables. Sakellariou et al. [13] just implemented an evacuation simulation where the evacuees are the agents with an emotional status instead of human psychological models. It is a subject of great interest that they referred to the emotion contagion.

In common, there is no proposal for evacuation simulation systems including agents with human psychological models in the above works. To construct a evacuation simulation system which reflect real human behaviors, agents have to be equipped with human psychological models.

\section{Proposed Model}

In order to make the evacuation simulation system for disasters more accurate, three psychological biases are proposed. One parameter is introduced to model the normalcy bias in each agent. Then the agents observe the other agents around him/her to decide the majority behavior that corresponds to the emotional contagion bias. Lastly the sympathy behavior bias is realized by applying roles to some agents.

\subsection{Normalcy Bias}

The role of the normalcy bias is to delay evacuation even if a disaster really occurs. For this, the parameter of "Level of Anxiety (LoA)" is incorporated in each agent as shown in Fig. 1. Once a disaster occurs, the LoA parameter monotonically increases each time a simulation step proceeds. Then the LoA parameter excesses a pre-defined threshold, the agent starts evacuation. The period between the disaster occurrence and the evacuation start corresponds to the situation under the normalcy bias. The initial value of the LoA parameter can be set as a different value for each agent. Thus it is possible to prepare various agent types from weak normalcy to strong normalcy. In simulation experiments, the initial values of the LoA parameter are provided based on some distribution.

\subsection{Emotional Contagion Bias}

As mentioned-above, the role of the emotional contagion bias is to let an agent follow to the other agents. For this purpose, we implement a model in which an agent recognizes the other agents around him/her. 


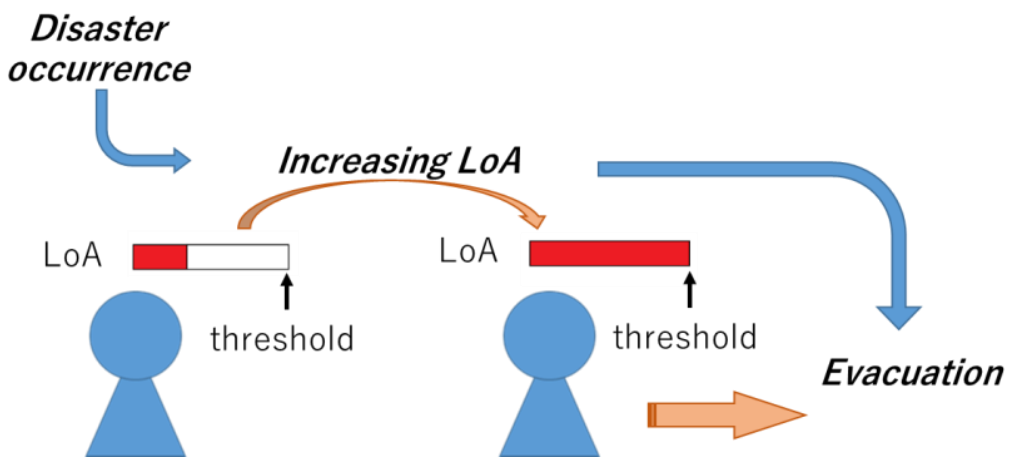

Fig. 1. Level of Anxiety (LoA) parameter for the nomalcy bias

As presented in Fig. 2, an agent (Agent A) can recognize the other agent (Agent B) within the area of circle with radius $r . r$ is a parameter of recognition distance. When Agent A recognizes Agent B, the LoA parameter decreases, and vice versa. It means both agents in the normalcy bias feel relieved more because of existence of another agent. Recognition occurs just once, while the LoA parameter increases according to the simulation step proceeding. As a result, when the LoA parameter attains to the threshold, the agent starts to evacuate.

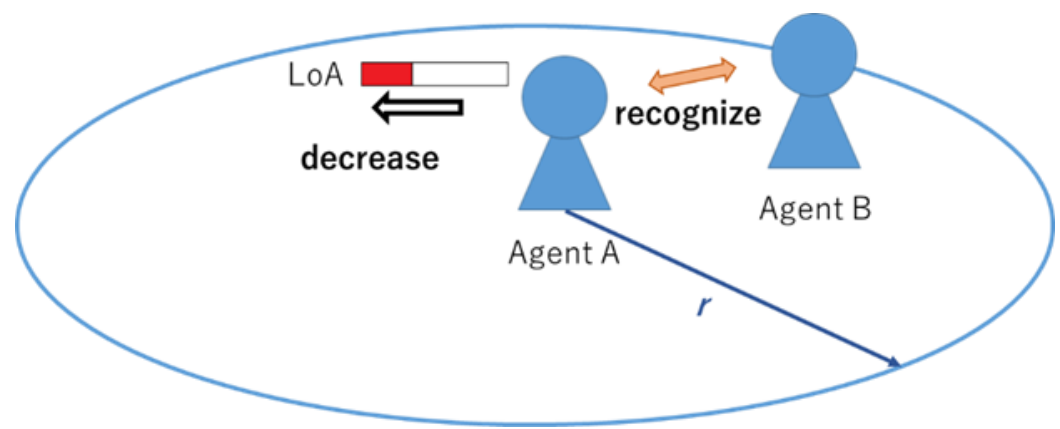

Fig. 2. A model to implement the emotional contagion bias

\subsection{Sympathy Behavior Bias}

As the targets of sympathy, elderly person agents are introduced. The characteris-tic of the elderly person agents is that their walking speed is slower than the nor-mal agents that are the agents referred in the previous sections. In addition, when a normal agent recognizes an elderly person agent within the area of the emotion-al contagion circle of the normal agent (Fig. 2), the normal agent slows down the walking speed to accompany with the elderly person agent. 


\section{$4 \quad$ Simulation Experimental Setting}

Aiming at urban disaster cases, an MAS with human psychological models has been implemented on a simulation system linked with GIS data. For the evalua-tion experiments, a virtual city area was selected for the simulation experiments. The size of the virtual city area that is shown in Fig. 3 is about $1.0 \mathrm{~km} \mathrm{X} 1.2 \mathrm{~km}$. In Fig. 3, thin lines running vertically and horizontally represent the roads where the agents have to walk for evacuation. The areas surrounded by the roads represent parks, buildings, and so on. As examples, an area of park and an area of building are directed in Fig. 3, although they may be difficult to be distinguished.

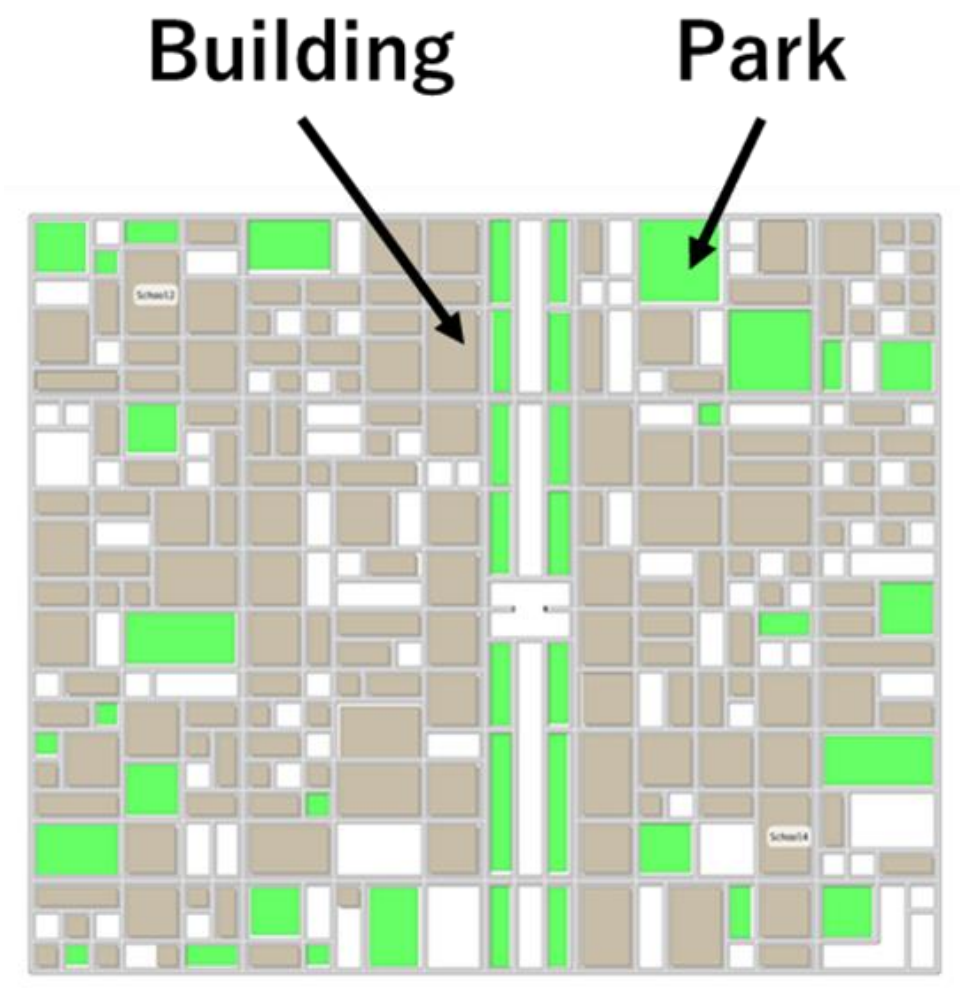

Fig. 3. The virtual city map used for simulation experiments

For the simulation experiments, two shelters that are the agents' evacuation goals are fixedly set at upper-left and lower-right points. The number of agents is 5,000, and all agents are assigned inside any building randomly at the start of each simulation experiment. It is assumed that each agent knows the shelter place which he/she aims to evacuate as the goal. 


\section{$5 \quad$ Simulation Results}

Using the experimental environment presented in Section 4, several simulation experiments were carried out to evaluate the proposed models.

\subsection{Simulation Results for Normalcy Bias}

Only the LoA parameter is set for each agent to evaluate effect of the normalcy bias modeling. In this simulation experiment, the initial distribution of the LoA parameter was changed. The lower the average of the LoA parameter, the stronger the effect of normalcy bias becomes. The threshold value of the LoA parameter, which is the limit value to start evacuation, was set to 360 .

The initial distribution of the LoA parameter was generated according to the uniform distribution. The range of the uniform distribution was changed as shown in Table 1 (b), (c), and (d). The agent model in Table1 (a) is a comparative model without implementing the LoA parameter.

Table 1. The agent models for normalcy bias simulation

\begin{tabular}{cc}
\hline & Agent models \\
\hline (a) & No normalcy bias \\
(b) & Normalcy bias only \\
& (Initial values: $360 \sim 240)$ \\
(c) & Normalcy bias only \\
& (Initial values: 300 180) \\
(d) & Normalcy bias only \\
& (Initial values: $240 \sim 120)$ \\
\hline
\end{tabular}

Figure 4 shows the experimental results for the agent models set as in Table 1 . The vertical axis presents the elapsed time to finish evacuation, that is to reach the pre-set evacuation goal. The horizontal axis presents the number of agents that completed evacuation. Therefore, when a graph reaches to 5,000 in the horizontal axis, the vertical axis means the time when all agents completed evacuation.

From Fig. 4, the agents finish evacuation earliest in the case without the normalcy bias. The stronger the normalcy bias becomes, the later all of the agents complete evacuation. The results correspond to our intuition and it shows that the simulation system successfully models the normalcy bias. 


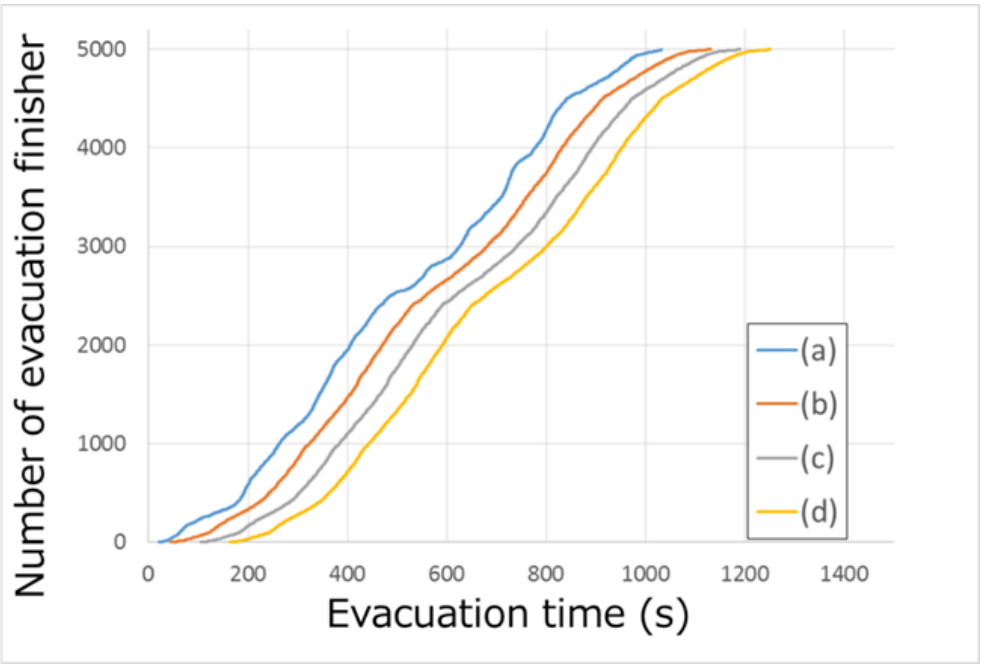

Fig. 4. The simulation results for the agent models in Table 1

\subsection{Simulation Results for Emotional Contagion Bias}

Next, the recognition function is implemented in the simulation system along with the LoA parameter. The recognition distance $r$ is set as $5 \mathrm{~m}$. Again, the initial distribution of the LoA parameter is the uniform distribution as in the experiment of Section 5.1. The ranges of the uniform distribution are shown in Table 2 (e), (f), and (g). Again, the agent model without the normalcy bias appears in Table2 (a) for comparison.

The experimental results for the agent models in Table 2 are presented in Fig. 5. The vertical and horizontal axes are the same as those in Fig. 4. From Fig. 5, the agent evacuation was delayed more than the cases in Fig. 4 because of the emotional contagion bias. It is also shown that the strength of the normalcy bias still has the evacuation delay.

Table 2. The agent models for emotional contagion bias simulation

\begin{tabular}{cc}
\hline & Agent models \\
\hline (a) & No normalcy bias \\
(e) & Normalcy bias only (Initial values: $360 \sim 240)$ \\
& + emotional contagion $(\mathrm{r}=5 \mathrm{~m})$ \\
(f) & Normalcy bias only (Initial values: $300 \sim 180)$ \\
& + emotional contagion $(\mathrm{r}=5 \mathrm{~m})$ \\
(g) & Normalcy bias only (Initial values: $240 \sim 120)$ \\
& + emotional contagion $(\mathrm{r}=5 \mathrm{~m})$
\end{tabular}




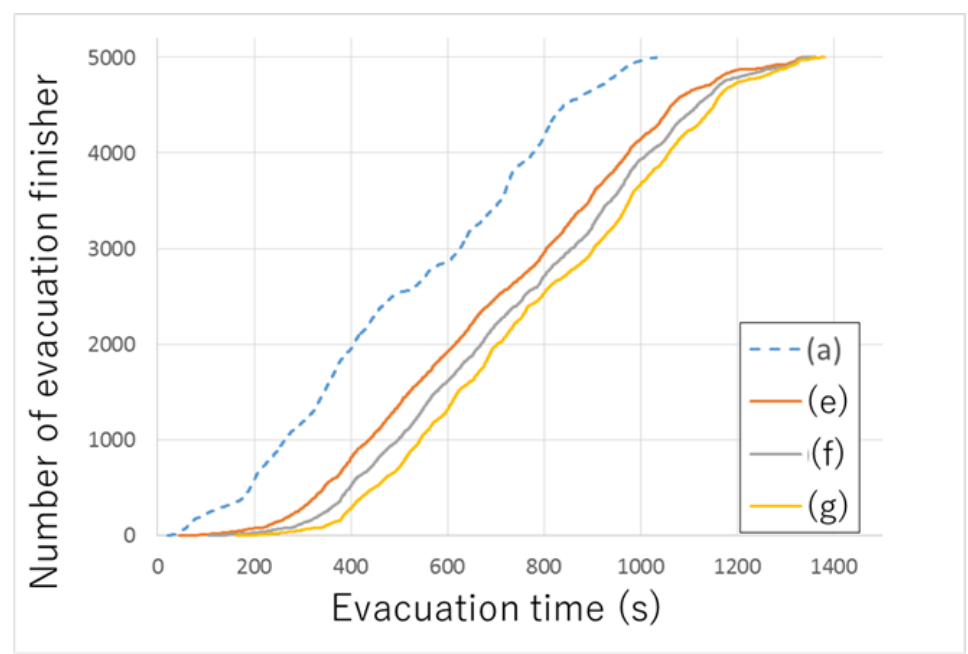

Fig. 5. The simulation results for the agent models in Table 2

\subsection{Simulation Results for Sympathy Behavior Bias}

Finally, the simulation experiments for the sympathy behavior bias were evaluated. In these experiments, the elderly person agents are introduced and two comparative cases are tested. The ratio of the elderly person agents was changed and the parameters are shown in Table 3 . Table 3 (h) shows the case where $25 \%$ of the elderly person agents are replaced for the normal agent. Similarly, Table 3 (i), (j), and (k) show the various cases in which the elderly person agents ratio changed when the agents have the normalcy bias. For comparison, the case with the normalcy bias is shown in Table 3 (d). For these simulation experiments, the range of the uniform distribution is the same as that in Table $1(\mathrm{~d})$.

Table 3. The agent models for sympathy behavior bias simulation

\begin{tabular}{cc}
\hline \multicolumn{1}{c}{ Agent models } \\
\hline (a) & No normalcy bias \\
(h) & No normalcy bias only + elderly persons (25\%) \\
(d) & Normalcy bias (Initial values: $240 \sim 120)$ \\
(h) & Normalcy bias only + elderly persons (10\%) \\
(j) & Normalcy bias only + elderly persons $(25 \%)$ \\
(k) & Normalcy bias only + elderly persons $(50 \%)$ \\
\hline
\end{tabular}

Figure 6 presents the results based on the model setting shown in Table 3. First of all, by comparing the cases (a) and (h), it can be said that evacuation completion time for all of the agents is almost the same for these two cases. Then we compare the cases 
of Table (d), (i), (j), and (k). Total evacuation time is almost the same for these four cases. From these experimental results, it is found that the sympathy behavior bias does not show any negative effect in evacuation time. The sympathy behavior is important to support the weak-side people such as the elderly persons. However, comparing the group of (a) and (h) and the group of (d), (i), (j), and (k), there is a gap between these two groups. From these results, it is found that the normalcy bias is a bottleneck to prevent fast-evacuation. We should educate people to remove the normalcy bias to accelerate their evacuation.

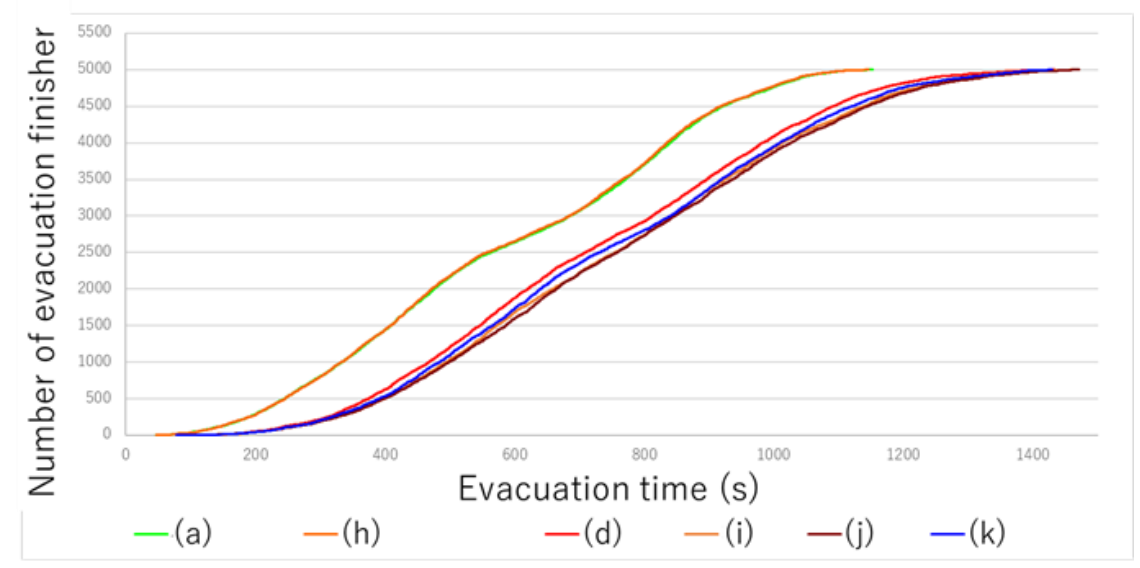

Fig. 6. The simulation results for the agent models in Table 3

\section{Conclusions}

In this paper, we have proposed the evacuation simulation models with the typi-cal psychological biases: the normalcy bias, the emotional contagion bias, and the sympathy behavior bias. These models have been implemented in an evacua-tion simulation system based on MAS and GIS for urban disaster situation. Sev-eral simulation experiments have been carried out and it is found that the pro-posed models are reasonable and correspond to our intuition.

The simulation model for evaluation was a virtual city area. Therefore, we are planning to implement these proposed models on the simulation system with a real GIS data. Moreover, other psychological models such as a straight walking bias should be considered to make the simulation system more realistic and more accurate.

It may be useful to learn actual human judgements and behavior from an evacuation drill in order to construct an evacuation model for simulations. For example, Jong and Helsloot [14] report that the flood risk awareness decreased through an exercise three days. Namely, the risk awareness was $21 \%$ on the first day, $18 \%$ on the second day and only $10 \%$ on the third day in the beginning. This awareness, however decreased on the first day with $9 \%$, while on the other days it increased with respectively $20 \%$ and $23 \%$. Although he situation is different that dealt in this research and the results could 
be different among drills, there must be something to be learned to empower the simulation models.

\section{References}

1. Ono, K. Civil-military Medical Assistance Cooperation after the Great East Japan Earthquake Experiences and Lessons. Center for Excellence in Disaster Management \& Humanitarian Assistance (COE-DMHA), Honolulu, LIAISON 5, 45-52 (2012).

2. Ferber, J.: Multi-Agent System: An Introduction to Distributed Artificial Intelligence. Addison-Wesley Professional, Boston (1999).

3. Vorst, H. C. M.: Evacuation Models and Disaster Psychology. In: First International Conference on Evacuation Modeling and Management, pp 15-21, (2010) doi: 10.1016/j.proeng.2010.07.004.

4. Campos, V., Bandeira, R., Bandeira, A.: A Method for Evacuation Route Planning in Disaster Situations. Procedia - Social and Behavioral Sciences 54, 503-512 (2012) doi: 1 0.1016/j.sbspro.2012.09.768

5. Stephens, N.M., Hamedani, M.-Y. G, Bergsieker, H.B., Eloul, L.: Why Did They " Choose”' to Stay? Perspectives of Hurricane Katrina Observers and Survivors. Psychological Science 20, 7, pp 878-886 (2009) doi: 10.1111/j.1467-9280.2009.02386.x

6. Ishii, S., Fujita, H., Mitsutake, M., Yamazaki, T., Matsuda, J., Matsuno, Y.: A Reinforcement Learning Scheme for a Partially-Observable Multi-Agent Game. Machine Learning 59 (1/2), 31-54, Springer, Heidelberg (2005).

7. Wu, S., Shuman, L., Bidanda, B., Balaban, C.: Agent-based Discrete Event Simulation Modeling for Disaster Responses. In: Industrial Engineering Research Conference. Fowler, J., Mason, S. (eds.), pp. 5-12, Society for Computer Simulation International (2008).

8. Chen, F., Zhai, Z., Madey, F.: Dynamic Adaptive Disaster Simulation: Developing a Predictive Model of. Emergency Behavior Using Cell Phone and GIS Data. In: Workshop on Agent-Directed Simulation (ADS '11), pp 5-12, Society for Computer Simulation International (2011).

9. Yun, J., Zhao, G., Fang, T., Liu, S., Huang C., Wang, C.: A Swarm-based Dynamic Evacuation Simulation Model Under the Background of Secondary Disasters. Systems Engineering Procedia 5, 61-67, Elsevier (2012) doi: 10.1016/j.sepro.2012.04.010

10. Samira, B., Fatima, B.: Multi-Agents Simulation of Human Behavior in a Situation of Emergency. Advanced Computing: An International Journal (ACIJ) 3 (1), 25-30 (2012) doi: 10.5121/acij.2012.3102

11. Mustaphaa, K., Mcheicka, H., Mellouli, S.: Modeling and Simulation Agent-Based of Natural Disaster Complex Systems. In: 4th International Conference on Emerging Ubiquitous Systems and Pervasive Networks (EUSPN-2013), Procedia Computer Science 21, 148-155 (2013).

12. Hofinger, G., Zinke, R., Künzer, L.: Human Factors in Evacuation Simulation, Planning, and Guidance. Transportation Research Procedia 2, 603-611, Elsevier (2014) doi: 10.1016/j.sepro.2012.04.010

13. Sakellariou, I., Kefalas, P., Stamatopoulou, I.: Evacuation Simulation through Formal Emotional Agent based Modelling In: 6th International Conference on Agents and Artificial Intelligence (ICAART 2014), pp 193-200 (2014).

14. Jong, M. de, Helsloot, I.: The Effects of Information and Evacuation Plans on Civilian Response During the National Dutch Flooding Exercise 'Waterproef'. Procedia Engineering, 3, pp 153-162, Elsevier (2010) doi: 10. .1016/j.proeng.2010.07.015 